\title{
OPEN Automated quantification of posterior vitreous inflammation: optical coherence tomography scan number requirements
}

\begin{abstract}
Jan Henrik Terheyden ${ }^{1 凶}$, Giovanni Ometto², Giovanni Montesano², Maximilian W. M. Wintergerst $\mathbb{1}^{1}$, Magdalena Langner ${ }^{1}$, Xiaoxuan Liu $\mathbb{C}^{3,4}$, Pearse A. Keane $\mathbb{( 1}^{5}$, David P. Crabb $\mathbb{1}^{2}$, Alastair K. Denniston $\mathbb{1}^{3,4,5}$ \& Robert P. Finger ${ }^{1 \bowtie}$

Quantifying intraocular inflammation is crucial in managing uveitis patients. We assessed the minimum B-scan density for reliable automated vitreous intensity (VI) assessment, using a novel approach based on optical coherence tomography (OCT). OCT volume scans centered on the macula were retrospectively collected in patients with uveitis. Nine B-scans per volume scan at fixed locations were automatically analyzed. The following B-scan selections were compared against the average score of 9 B-scans per volume scan as a reference standard: $1 / 3 / 5 / 7$ central scans $(1 c / 3 c / 5 c / 7 c), 3$ widely distributed scans ( $3 \mathrm{w}$ ). Image data of 49 patients ( 31 females) were included. The median VI was 0.029 (IQR: 0.032). The intra-class-correlation coefficient of the VI across the 9 B-scans was 0.923. The median difference from the reference standard ranged between 0.001 (7c) and 0.006 (1c). It was significantly lower for scan selection $3 w$ than $5 c$, $p$ (adjusted) $=0.022$, and lower for selection $7 c$ than $3 w, p$ (adjusted) $=0.003$. The scan selections $7 c$ and $3 w$ showed the two highest areas under the receiver operating curve $(0.985$ and 0.965 , respectively). Three widely distributed B-scans are sufficient to quantify VI reliably. Highest reliability was achieved using 7 central B-scans. Automated quantification of $\mathrm{VI}$ in uveitis is reliable and requires only few OCT B-scans.
\end{abstract}

Uveitis is a common inflammatory disease of the eye, accounting for 5-10\% of visual impairment worldwide ${ }^{1,2}$. The disease affects the vascular layer (consisting of iris, ciliary body and choroid) of people who are frequently of working age $\mathrm{e}^{1,3}$. Quantification of intraocular inflammation is crucial in managing patients with uveitis. To date the quantification of intraocular inflammation is mostly done semi-quantitatively by subjective clinical evaluation, which comes with a range of limitations common to subjective ratings ${ }^{3-5}$. Thus, several approaches have been developed to quantify vitreous intensity (VI) more objectively ${ }^{6-9}$. This includes quantification of vitreous inflammation based on optical coherence tomography (OCT) scans ${ }^{8-12}$.

The developed algorithm for an automatic assessment of vitreous inflammation is based on the measurement of hyperreflective spots within the posterior vitreous included on macular OCT scans. As this parameter alone is prone to artefacts due to media opacities, a score relative to the retinal pigment epithelium has been established in previous studies and evaluated against the reference standard of the Standardization of Uveitis Nomenclature (SUN) clinical grading of vitreous haze $\mathrm{e}^{8-13}$.

The application of an OCT-based, automated algorithm for quantification of vitreous inflammation requires manual selection and a certain amount of manual post-processing steps of scans. For this reason, the number of scans should be limited to the minimum amount required for reliable quantification of VI to facilitate future employment in clinical routine and randomized controlled clinical trials. These applications include a potential use of the OCT-based parameter as a biomarker for therapeutic decisions, follow-up intervals and as a clinical

\footnotetext{
${ }^{1}$ Department of Ophthalmology, University Hospital Bonn, 53127 Bonn, Germany. ${ }^{2}$ Division of Optometry and Visual Science, City, University of London, London, UK. ${ }^{3}$ Academic Unit of Ophthalmology, Institute of Inflammation and Ageing, University of Birmingham, Birmingham, UK. ${ }^{4}$ Department of Ophthalmology, University Hospitals Birmingham NHS Foundation Trust, Birmingham, UK. ${ }^{5} \mathrm{NIHR}$ Biomedical Research Centre At Moorfields Eye Hospital and UCL Institute of Ophthalmology, London, UK. ${ }^{\bowtie}$ email: Jan.Terheyden@ukbonn.de; Robert.Finger@ukbonn.de
} 

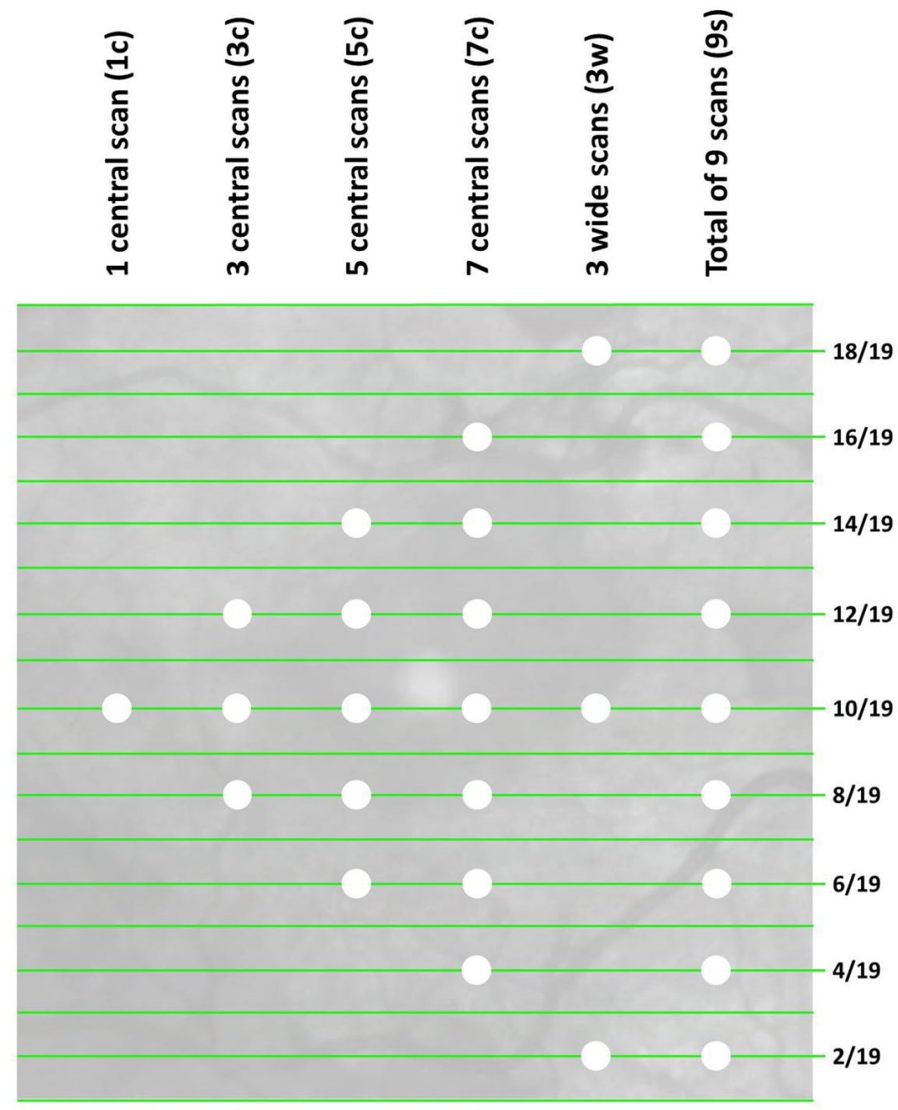

Figure 1. Illustration of an infrared image linked to an OCT volume scan that consists of $19 \mathrm{~B}$-scans. The white dots indicate which scans (green lines) have been included in the different sub selections of B-scans (columns).

\begin{tabular}{|l|l|l|}
\hline Scan sub selection & $\begin{array}{l}\text { Median VI difference from reference standard } \\
\text { (interquartile range) }\end{array}$ & Limits of agreement compared to reference standard \\
\hline 1 central scan $(1 \mathrm{c})$ & $0.006(0.009)$ & {$[-0.039 ; 0.037]$} \\
\hline 3 central scans $(3 \mathrm{c})$ & $0.005(0.011)$ & {$[-0.033 ; 0.032]$} \\
\hline 5 central scans $(5 \mathrm{c})$ & $0.004(0.009)$ & {$[-0.028 ; 0.026]$} \\
\hline 7 central scans $(7 \mathrm{c})$ & $0.001(0.004)$ & {$[-0.009 ; 0.009]$} \\
\hline 3 wide scans $(3 \mathrm{w})$ & $0.003(0.005)$ & {$[-0.014 ; 0.013]$} \\
\hline
\end{tabular}

Table 1. Deviation between the reference standard (VI means of 9 B-scans) and average VI values from the sub selections of B-scans and respective limits of agreement. VI, vitreous intensity.

trial endpoint. Thus, we assessed the minimum required number of B-scans to reliably quantify vitreous inflammation in this study.

\section{Results}

Current image data of 49 eyes of 49 patients ( 31 females, 18 males) examined at a tertiary referral centre were included. Uveitis was classified as intermediate in 8 eyes, posterior in 33 eyes and panuveitis in 8 eyes. Mean age at examination was $70 \pm 12$ years; mean $\log$ MAR BCVA at examination was $0.5 \pm 0.3$ and 44 eyes were pseudophakic.

The mean distance between two B-scans was $243 \pm 8 \mu \mathrm{m}$ (Fig. 1, individual B-scans are represented by green lines). Across all B-scans, the median VI was 0.029 (interquartile range: 0.032 ), ranging from 0.0026 to 0.394 . The mean VI per eye did not differ significantly between phakic and pseudophakic eyes $(P=0.919)$. The intra-class correlation coefficient of the VI values across the 9 B-scans was 0.923 ( $95 \%$ confidence interval $0.886-0.952$ ), indicating high agreement between VI values.

Smaller median differences indicate less variation from the chosen reference standard (i.e. the mean VI value from 9 B-scans, Fig. 1). Table 1 shows that the median differences between the reference standard and the average values of scan selections ( 1 central scan, $1 c$; 3 central scans, $3 c ; 5$ central scans, $5 c ; 7$ central scans, $7 c$ and 3 widely distributed scans, $3 \mathrm{w}$ ) were noticeably different. For instance, the difference from the total VI average in 


\begin{tabular}{|l|l|}
\hline Scan sub selection & AUC $[\mathbf{9 5 \% ~ C I ] ~}$ \\
\hline 1 central scan $(1 c)$ & $0.862[0.753 ; 0.971]$ \\
\hline 3 central scans $(3 c)$ & $0.870[0.767 ; 0.974]$ \\
\hline 5 central scans $(5 c)$ & $0.929[0.855 ; 1.0]$ \\
\hline 7 central scans $(7 c)$ & $0.985[0.954 ; 1.0]$ \\
\hline 3 wide scans $(3 w)$ & $0.965[0.912: 1.0]$ \\
\hline
\end{tabular}

Table 2. Area under the curve values for the detection of values larger than the dataset's median, per scan sub selection. AUC, area under the curve; CI, confidence interval.

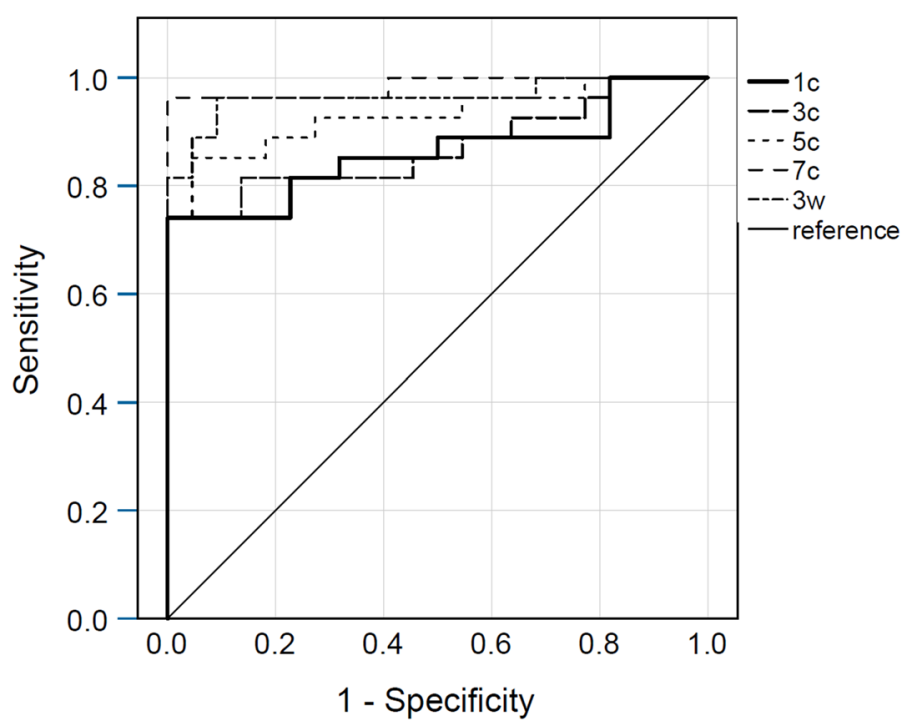

Figure 2. Receiver operating curves of the different scan sub selections. The state variable was the dataset's median. 1c, 1 central scan; $3 c, 3$ central scans; $5 c, 5$ central scans; $7 c, 7$ central scans; $3 w$, 3 wide scans.

$9 \mathrm{~B}$-scans was smaller in the sub selection $3 \mathrm{w}$ compared to $5 \mathrm{c}$ (Holm-Bonferroni adjusted $P=0.022$ ). It was also smaller in the sub selection $7 \mathrm{c}$ compared to $3 \mathrm{w}$ (Holm-Bonferroni adjusted $P=0.003$ ).

Linear regression analysis revealed no significant associations between the two axes on Bland-Altman plots with each other, comparing the reference standard with the VI scores from individual scan selections (comparators: $1 \mathrm{c}, \mathrm{p}=0.907 ; 3 \mathrm{c}, \mathrm{p}=0.120 ; 5 \mathrm{c}, \mathrm{p}=0.172 ; 7 \mathrm{c}, \mathrm{p}=0.604 ; 3 \mathrm{w}, \mathrm{p}=0.243)$.

All area under the curve (AUC) values from ROC analysis were $>0.8$ (Table 2), indicating high sensitivity and specificity for the detection of values larger than the dataset's median, i.e. detection of eyes with statistically "higher inflammation" in contrast to eyes with "lower inflammation". The scan sub selections 7c and 3w achieved the highest sensitivity and specificity values (Fig. 2). We achieved similar results using a comparison with the dataset's upper and lower quartile as state variables (data not shown).

\section{Discussion}

The results indicate that Vitreous/RPE-relative intensity is consistent across OCT scans in individuals with uveitis. Averaging the VI of several B-scans within one OCT volume scan further improved measurement reliability because it reduces the influence of local structural alterations. VI calculation from three widely distributed B-scans (average distance $1944 \mu \mathrm{m}$ ) achieves comparable results with VI calculation from nine equally distributed B-Scans (average distance $486 \mu \mathrm{m}$ ) and allows for sufficient discrimination of different levels of inflammation.

Our results indicate that less dense scan patterns compare well to more dense scans in the quantification of VI. As the biomarker itself as well as the measurement of vitreous inflammation using OCT are relatively novel, no data comparing different scan densities for this purpose are available so far. However, different OCT scan patterns of the macula have been compared in the literature with respect to detection of retinal biomarkers such as the presence of intraretinal fluid and quantification of retinal layer thicknesses. Sayanagi and colleagues did not find a significant difference in retinal thickness measurements of patients with macular diseases between a dense OCT volume scan and a radial scan consisting of 6 lines ${ }^{14}$. Other groups confirmed that retinal thickness can be measured almost as reliably with low B-scan density scans compared to high density scans in individuals with defined macular diseases such as diabetic macular oedema ${ }^{15,16}$, age-related macular degeneration ${ }^{17}$ and retinal vein occlusion ${ }^{18}$. Different studies showed the detection of fluid to be almost as sensitive in scan patterns 
only $25-50 \%$ as dense as the respective reference standard in age-related macular degeneration, diabetic macular oedema, retinal vein occlusion and other retinal disease ${ }^{17-23}$.

Our main result that a pattern with a smaller number of OCT B-scans-based measurements is similarly sensitive as a denser reference standard is thus consistent with findings reported in the literature. The scan sub selection " $3 \mathrm{w}$ " including a central B-scan and two peripheral B-scans was superior to a single central scan, 3 central scans and 5 central scans. Reliability can be increased with 7 B-scans, i.e. the difference from the reference standard was significantly lower, but the relevance of this small decrease in mean difference is unclear and needs to be considered against the increased workload.

Of note is that the minimum scan density that can be recommended for the measurement of VI is lower than the one recommended for use in retinal diseases to assess retinal thickness or presence of macular oedema reported in the literature. The minimum number of B-scans required for these purposes varies between five and 32 scans $^{17-23}$. The diffuse nature of the signal in the vitreous cavity in inflammatory diseases in contrast to clearly locatable pathologies in only a small part of the retina in retinal diseases might be a potential explanation for this. However, the impact of local heterogeneity in vitreous haze or accumulation of inflammatory cells (e.g. snowballs) on the OCT-based parameter and its changes with eye movement require further investigation.

The strengths of our study include a standardized imaging protocol for all participants, a relatively homogenous sample consisting of only uveitis patients (excluding anterior uveitis) and the use of a previously developed algorithm which is already clinically validated. Limitations include the relatively small sample size with only a limited number of B-scans per subject available, the use of only one device (Spectralis, Heidelberg Engineering), the relatively high age of the participants for a uveitis population, and the limited availability of clinical data. In this study we have considered measurements taken at a single visit, and therefore have not considered the stability of the signal over time.

Overall, our study shows that automated determination of VI is reliable across OCT B-scans in uveitis patients. The recommended minimum B-scan density for future research based on this parameter is three horizontal scans: One central scan and two peripheral scans located approximately $2000 \mu \mathrm{m}$ inferiorly and superiorly from the central B-scan ( $3 \mathrm{w})$. Measurements were even more stable across scans in a pattern of 7 horizontal scans $(7 \mathrm{c})$ but we interpret this improvement as not clinically relevant compared to the recommended pattern. In the future, further correlation of the data with clinical vitreous haze scores and other clinical variables as well as further reliability analysis based on these values is warranted.

\section{Methods}

The retrospective study took place at the department of ophthalmology of the University of Bonn, Germany. The institutional Ethics Committee (University Hospital Bonn, Germany) approved the use of retrospective data for study purposes and approved that informed consent can be waived due to the use of retrospective data only (no. 103/18). The study adhered to the principles of the declaration of Helsinki. Participants were included if they had a form of Posterior Segment Involving Uveitis (PSIU) i.e. one of Intermediate, posterior or panuveitis as classified according to the SUN criteria ${ }^{3}$.

Image data. Macular OCT volume scans were retrospectively collected. OCT is a light-based, non-invasive technique frequently applied in ophthalmology. It is based on local interference between two signals (object signal and reference signal $)^{24,25}$. Using software, B-scans (e.g. axial) are automatically calculated from A-scans. Retinal OCT B- scans show parts of the posterior vitreous cavity, the retinal layers as well as choroidal structures. The volume scans were obtained with the Spectralis SD-OCT (Heidelberg Engineering, Heidelberg, Germany), with a B-scan image resolution of $512 \times 496$ pixels and 5 images averaged (automated real-time tracking mode $=5$ ). Inclusion criteria were volume scans consisting of $19 \mathrm{~B}$-scans each and a B-scan size of $20^{\circ} \times 15^{\circ}$. Exclusion criteria were insufficient image quality (HEYEX software image quality score $<20$ in $>3$ B-scans), incomplete scan, fixation errors and a presumed disease aetiology other than uveitis. Besides image data, age, uveitis classification, best-corrected visual acuity (BCVA) and lens status of all included patients were collected.

Image analysis. Every other B-scan was selected from the OCT volume scans, resulting in 9 B-scans per volume scan available for analysis (Fig. 1). As one of the previous VI algorithm validation studies included a reference of $7 \mathrm{~B}$-scans per volume, we used a comparable density as our gold standard ${ }^{11}$. The image data and additional image acquisition parameters were imported into MATLAB, Version R2016a (The MathWorks, Natick, Massachusetts, USA). The VI parameter Vitreous/Retinal Pigment Epithelium (RPE)-relative intensity was automatically calculated per B-scan according to an algorithm that has previously been described and clinically validated ${ }^{8-12,26}$. In summary, pre-processing steps include opening, thresholding and adjustment as outlined by Keane et al. ${ }^{9}$. The posterior part of the vitreous cavity is automatically detected and the OCT sum signal in this area is quantified relative to the RPE signal intensity in order to lower the impact of media opacities on the outcome parameter. The overall vitreous reflectivity is increased in inflammation which has been explained e.g. by inflammatory components and proteins in the vitreous cavity ${ }^{9,26}$. B-scan quality was assessed for all selected $\mathrm{B}$-scans and the distance between B-scans was obtained per individual volume scan.

Statistical analyses. The intra-class correlation coefficient between all VI values per volume scan was calculated. The single VI value of the central B-scan (1c) and averaged VI values of five combinations of B-scans ( 3 , 5 and 7 central scans $(3 c, 5 c, 7 c)$, all 9 scans available for analysis $(9 \mathrm{~s}), 3$ widely distributed scans ( $3 w)$; Fig. 1) were computed for all volume scans included. The averaged VI value of $9 \mathrm{~B}$-scans was used as the standard reference. Mean absolute differences between this reference and a single central B-scan VI as well as the averaged VI values listed above $(3 c, 5 c, 7 c, 3 w)$ were calculated. $95 \%$ limits of agreement (LoA) were calculated according to 
the formula LoA $=$ mean $\pm 1.96 \times$ standard deviation of the differences between the two measurements. Linear regression analysis was performed based on Bland-Altman plots to identify associations between the above mentioned mean absolute differences (e.g. $9 \mathrm{~s}-1 \mathrm{c}, 9 \mathrm{~s}-3 \mathrm{c}, 9 \mathrm{~s}-5 \mathrm{c}$, etc.) and their respective means, excluding four cases that were likely outside of the sensitivity range of our study (mean VI score $>0.1$ ). In addition, we performed receiver operating curve characteristic (ROC) analysis for discrimination of VI values greater or equal and VI values smaller than the median VI value out of all B-scans.

Statistical analyses were performed with SPSS Statistics, version 25 (IBM Corporation, Armonk, New York, USA) and R, version 3.5.0 (R Core Team, Vienna, Austria). Paired samples were compared with the Wilcoxon rank sum test correcting for multiple comparisons using the Holm-Bonferroni method ${ }^{27}$. The level of statistical significance was $P<0.05$.

Received: 26 May 2020; Accepted: 25 January 2021

Published online: 08 February 2021

\section{References}

1. Miserocchi, E., Fogliato, G., Modorati, G. \& Bandello, F. Review on the worldwide epidemiology of uveitis. Eur. J. Ophthalmol. 23, 705-717 (2013).

2. Tsirouki, T. et al. A focus on the epidemiology of uveitis. Ocul. Immunol. Inflamm. 26, 2-16 (2018).

3. Standardization of Uveitis Nomenclature (SUN) Working Group. Standardization of uveitis nomenclature for reporting clinical data Results of the First International Workshop. Am. J. Ophthalmol. 140, 509-516 (2005).

4. Kempen, J. H., Ganesh, S. K., Sangwan, V. S. \& Rathinam, S. R. Interobserver agreement in grading activity and site of inflammation in eyes of patients with uveitis. Am. J. Ophthalmol. 146, 813-818.e1 (2008).

5. Davis, J. L. et al. Scale for photographic grading of vitreous haze in uveitis. Am. J. Ophthalmol. 150, 637-641.e1 (2010).

6. Tugal-Tutkun, I. \& Herbort, C. P. Laser flare photometry: a noninvasive, objective, and quantitative method to measure intraocular inflammation. Int. Ophthalmol. 30, 453-464 (2010).

7. Herbort, C. P., Guex-Crosier, Y., de Ancos, E. \& Pittet, N. Use of laser flare photometry to assess and monitor inflammation in uveitis. Ophthalmology 104, 64-71 (1997) (discussion 71-2).

8. Keane, P. A. et al. Objective measurement of vitreous inflammation using optical coherence tomography. Ophthalmology 121, 1706-1714 (2014).

9. Keane, P. A. et al. Automated analysis of vitreous inflammation using spectral-domain optical coherence tomography. Transl. Vis. Sci. Technol. 4, 4 (2015).

10. Sreekantam, S. et al. Quantitative analysis of vitreous inflammation using optical coherence tomography in patients receiving sub-Tenon's triamcinolone acetonide for uveitic cystoid macular oedema. Br. J. Ophthalmol. 101, 175-179 (2017).

11. Montesano, G. et al. Optimizing OCT acquisition parameters for assessments of vitreous haze for application in uveitis. Sci. Rep. 8, 1-7 (2018).

12. Zarranz-Ventura, J. et al. Evaluation of objective vitritis grading method using optical coherence tomography: influence of phakic status and previous vitrectomy. Am. J. Ophthalmol. 161, 172-80.e1-4 (2016).

13. Coric, D. et al. Objective quantification of vitreous haze on optical coherence tomography scans: no evidence for relationship between uveitis and inflammation in multiple sclerosis. Eur. J. Neurol. 27, 144-e3 (2020).

14. Sayanagi, K., Sharma, S. \& Kaiser, P. K. Comparison of retinal thickness measurements between three-dimensional and radial scans on spectral-domain optical coherence tomography. Am. J. Ophthalmol. 148, 431-438 (2009).

15. Nittala, M. G., Konduru, R., Ruiz-Garcia, H. \& Sadda, S. R. Effect of OCT volume scan density on thickness measurements in diabetic macular edema. Eye (London) 25, 1347-1355 (2011).

16. Taban, M., Sharma, S., Williams, D. R., Waheed, N. \& Kaiser, P. K. Comparing retinal thickness measurements using automated fast macular thickness map versus six-radial line scans with manual measurements. Ophthalmology 116, 964-970 (2009).

17. Velaga, S. B. et al. Impact of optical coherence tomography scanning density on quantitative analyses in neovascular age-related macular degeneration. Eye (London) 31, 53-61 (2017).

18. Rahimy, E., Rayess, N., Maguire, J. I. \& Hsu, J. Radial versus raster spectral-domain optical coherence tomography scan patterns for detection of macular pathology. Am. J. Ophthalmol. 158, 345-353.e2 (2014).

19. Fang, P. P. et al. Minimal optical coherence tomography B-scan density for reliable detection of intraretinal and subretinal fluid in macular diseases. Retina 39, 150-156 (2019).

20. Baranano, A. E., Keane, P. A., Ruiz-Garcia, H., Walsh, A. C. \& Sadda, S. R. Impact of scanning density on spectral domain optical coherence tomography assessments in neovascular age-related macular degeneration. Acta Ophthalmol. 90, e274-e280 (2012).

21. Sadda, S. R., Keane, P. A., Ouyang, Y., Updike, J. F. \& Walsh, A. C. Impact of scanning density on measurements from spectral domain optical coherence tomography. Investig. Ophthalmol. Vis. Sci. 51, 1071-1078 (2010).

22. de Niro, J. E. et al. Sensitivity of fluid detection in patients with neovascular and using spectral domain optical coherence tomography high-definition line scans. Retina 34, 1163-1166 (2014).

23. Adam, M. K., Rayess, N., Rahimy, E., Maguire, J. I. \& Hsu, J. Radial versus raster spectral-domain optical coherence tomography scan patterns for detection of macular fluid in neovascular age-related macular degeneration. Br. J. Ophthalmol. 100, 491-494 (2016).

24. Podoleanu, A. G. Optical coherence tomography. J. Microsc. 247, 209-219 (2012).

25. Huang, D. et al. Optical coherence tomography. Science (New York, N.Y.) 254, 1178-1181 (1991),

26. Denniston, A. K., Keane, P. A. \& Srivastava, S. K. Biomarkers and surrogate endpoints in uveitis: the impact of quantitative imaging. Investig. Ophthalmol. Vis. Sci. 58, BIO131-BIO140 (2017).

27. Holm, S. A simple sequentially rejective multiple test procedure. Scand. J. Statist. 6, 65-70 (1979).

\section{Acknowledgments}

This work was supported by the BONFOR GEROK Program, Faculty of Medicine, University of Bonn, Grant No O-137.0028 to MWMW.

\section{Author contributions}

J.H.T. and R.P.F. designed the study, G.O., G.M., X.L., P.A.K. and A.K.D. created the software used in this work, G.O., J.H.T. and M.L. acquired and analysed the data. J.H.T., M.W.M.W. and R.P.F. interpreted the data, J.H.T. and R.P.F. drafted the manuscript, G.O., G.M., M.W.M.W., M.L., X.L., P.A.K., D.P.C. and A.K.D. substantively revised the manuscript. All authors have approved the submitted version and have agreed both to be personally accountable for the author's own contributions and to ensure that questions related to the accuracy or integrity of 
any part of the work, even ones in which the author was not personally involved, are appropriately investigated, resolved, and the resolution documented in the literature.

\section{Funding}

Open Access funding enabled and organized by Projekt DEAL. This research was supported by funding of the German Scholars Organization/Else Kröner Fresenius Stiftung (GSO/EKFS 16) to RF, by BONFOR GEROK Program, Faculty of Medicine, University of Bonn, Grant No. O-137.0028 to MWMW and by a Wellcome Trust Health Innovation Challenge grant (200141/Z/15/Z) to XL, GO, AKD, DPC and PAK.

\section{Competing interests}

The Department of Ophthalmology, University of Bonn received imaging devices from Heidelberg Engineering, Optos, Carl Zeiss Meditec, and CenterVue. JHT: No additional conflicts of interest. GO: None. GM: None. MWMW: Travel grant/reimbursement from DigiSight Technologies, Heine Optotechnik GmbH, ASKIN \& CO $\mathrm{GmbH}$ and Berlin-Chemie AG, grants from Heine Optotechnik and Berlin-Chemie AG, consultant for Heine Optotechnik GmbH, imaging devices from DigiSight Technologies, D-EYE Srl and Heine Optotechnik GmbH, free analysis from Eyenuk Inc. ML: No additional conflicts of interest. XL: None. PAK: Grants from Allergan, Bayer, Carl Zeiss Meditec, Haag-Streit, Heidelberg Engineering, Novartis, Topcon, consultant for Deepmind. AKD: None. DPC: Speaker fees from Allergan, Bayer, Santen; unrestricted funding from Allergan, Santen; consultancy with Centervue-all outside the remit of the submitted work. RPF: Consultant for Bayer, Novartis, Santen, Opthea, Novelion, Santhera, Inositec, Alimera and RetinaImplant; honoraria from Bayer, Ellex, Alimera.

\section{Additional information}

Correspondence and requests for materials should be addressed to J.H.T. or R.P.F.

Reprints and permissions information is available at www.nature.com/reprints.

Publisher's note Springer Nature remains neutral with regard to jurisdictional claims in published maps and institutional affiliations.

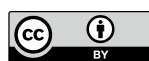

Open Access This article is licensed under a Creative Commons Attribution 4.0 International License, which permits use, sharing, adaptation, distribution and reproduction in any medium or format, as long as you give appropriate credit to the original author(s) and the source, provide a link to the Creative Commons licence, and indicate if changes were made. The images or other third party material in this article are included in the article's Creative Commons licence, unless indicated otherwise in a credit line to the material. If material is not included in the article's Creative Commons licence and your intended use is not permitted by statutory regulation or exceeds the permitted use, you will need to obtain permission directly from the copyright holder. To view a copy of this licence, visit http://creativecommons.org/licenses/by/4.0/.

(C) The Author(s) 2021 